\title{
Fett ist Gift für Herz und Hirn
}

\author{
Chefredakteur Dr. med. Dirk Einecke
}

\section{APFELBAUCH GEFÄHRDET GEHIRN}

\section{Erst dick, dann dement}

Unabhängig von Diabetes und kardiovaskulären Erkrankungen erhöht Adipositas das Demenzrisiko. Im Rahmen einer Longitudinalstudie waren bei 6583 Probanden im mittleren Lebensalter in den Jahren 1964-1973 der Bauchumfang und der BMI gemessen worden. 36 Jahre später hatten 1049 Studienteilnehmer eine Demenz entwickelt. Besonders häufig betroffen waren diejenigen, die in der Lebensmitte eine ausgeprägte Stammfettsucht aufgewiesen hatten. Auch bei normalem BMI erhöhte ein dicker Bauch das Demenzrisiko um über $80 \%$. Bei Personen mit bauchbetontem Übergewicht

\section{PRÄVENTION}

\section{Hochdruck liegt in der Familie}

Kinder von Eltern mit Bluthochdruck haben ein erhöhtes Risiko, später auch eine Hypertonie zu entwickeln. Besonders groß ist dieses Risiko, wenn beide Eltern schon vor dem 55. Lebensjahr erkrankt sind: Ein Hochdruck bildet sich dann rund sechsmal häufiger als bei Kindern von Eltern mit normalen Blutdruckwerten. Das zeigt die Auswertung von Daten der Johns

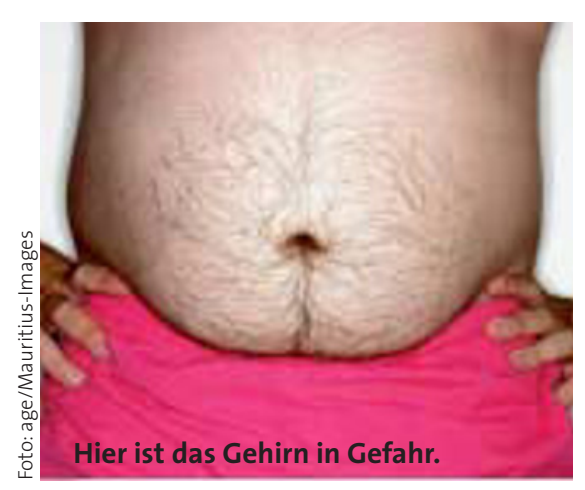

oder Adipositas war das Risiko 2,34-bzw. 3,6-mal höher als bei Normalgewichtigen ohne „Schwimmreifen“.

Neurology 2008, doi: 10.1212/o1.wnl.0000306313. 89165.ef
Hopkins Precursors Study, bei denen der Blutdruck von 1160 Männern über einen Zeitraum von 50 Jahren protokolliert wurde. Die Studienautoren raten deswegen, bei Patienten mit entsprechender familiärer Belastung schon in jungen Jahren den Blutdruck im Auge zu behalten und präventive Maßnahmen zu ergreifen. Arch Intern Med 2008;168:643-648

\section{ALTERSBEDINGTER MUSKELSCHWUND}

\section{Bei Frauen schwerer zu kompensieren}

Ab etwa dem 50. Lebensjahr verliert der Mensch bis zu 0,4\% seiner Muskelmasse pro Jahr. Frauen haben weniger Muskeln als Männer, dafür schreitet der Abbau an Muskelmasse bei ihnen langsamer voran. Dies ergaben Untersuchungen an 29 gesunden Probanden im Alter zwischen 65 und 80 Jahren. Für Frauen ist es aber schwerer, dem Muskelabbau entgegenzuwirken. Es wird deswegen empfohlen, dass sie sich im Alter möglichst proteinreich ernähren.

http://www.plosone.org/article/info\%3Adoi\%2F10. 1371\%2Fjournal.pone.0001875

\section{Zitat der Woche}

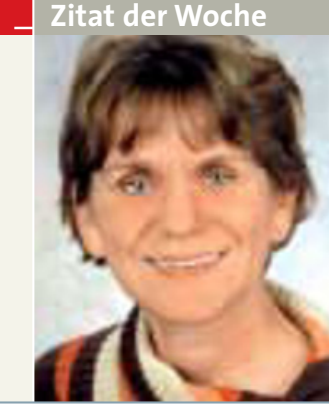

„Die Erblindung ist die Komplikation, vor der Diabetiker am meisten Angst haben. Deshalb verschließen sie oft die Augen davor."

Diana Drossel vom Deutschen Blinden- und Sehbehindertenverband e. V., Eschweiler, beim Kongress der Augenärztlichen Akademie Deutschland vom 4.-8. März in Düsseldorf

\section{SEKUNDÄRPRÄVENTION DER KHK}

\section{Auch bei alten Patienten lebensverlängernd}

Die Sterblichkeit von Postinfarktpatienten ist einer US-amerikanischen Registerstudie zufolge von 1995 bis 2004 um $3 \%$ pro Jahr gesunken. Die längere Überlebenszeit wird vor allem auf die verbesserte medikamentöse Sekundärprävention zurückgeführt. Damit bestätigt sich, dass sich der in klinischen Studien nachgewiesene Nutzen dieser Therapien auch auf „reale“ Patienten erstreckt. Diese waren mit durchschnittlich 80 Jahren deutlich älter als die meisten Studienpopulationen. Die leitliniengemäße Sekundärprävention einer KHK sollte daher auch bei betagten Patienten angestrebt werden.

J Am Coll Cardiol 2008;51:1247-1254

\section{SOZIALISATION}

\section{Einsame Kinder werden depressive Erwachsene}

Wenn Kinder nicht in der Lage sind, soziale Kontakte zu knüpfen, ist das Risiko groß, dass sie in der Adoleszenz zu Depressionen und Angsterkrankungen neigen. Dies ergab eine Studie, die 205 Probanden in ihrer Entwicklung vom achten bis zwölften Lebensjahr bis ins frühe Erwachsenenalter verfolgte.

Child Development 2008;79:359

\section{LANGZEITSTUDIE}

\section{Die späten Folgen einer frühzeitigen Geburt}

Anhand des norwegischen Geburtenregisters der Jahre 1967-1988 wurde die Prognose von Frühchen untersucht. Dabei zeigte sich, dass die frühe Geburt die Sterblichkeit im Kindesalter erhöht: Bei einer Geburt vor der 28. Schwangerschaftswoche waren solche Todesfälle fünf- bis zehnmal häufiger. Frauen und Männer, die als Frühchen zur Welt gekommen waren, hatten außerdem seltener eigene Kinder.

JAMA 2008;299:1429-1436 